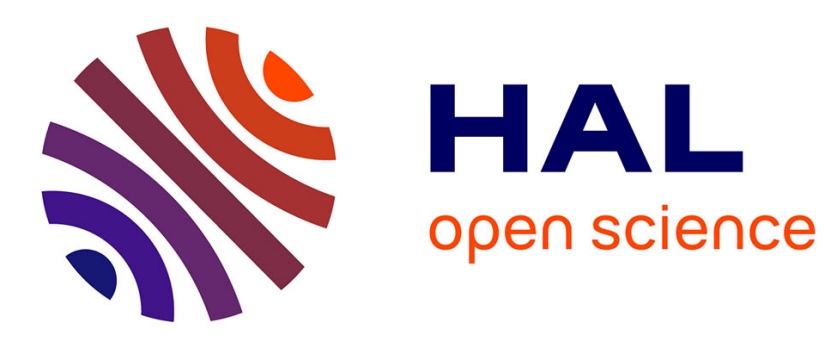

\title{
A Solution to a Problem of D. Lau: Complete Classification of Intervals in the Lattice of Partial Boolean Clones
}

Miguel Couceiro, Lucien Haddad, Karsten Schölzel, Tamas Waldhauser

\section{- To cite this version:}

Miguel Couceiro, Lucien Haddad, Karsten Schölzel, Tamas Waldhauser. A Solution to a Problem of D. Lau: Complete Classification of Intervals in the Lattice of Partial Boolean Clones. Journal of Multiple-Valued Logic and Soft Computing, 2017, 28 (1), pp.47-58. hal-01183004

\section{HAL Id: hal-01183004 \\ https://inria.hal.science/hal-01183004}

Submitted on 5 Aug 2015

HAL is a multi-disciplinary open access archive for the deposit and dissemination of scientific research documents, whether they are published or not. The documents may come from teaching and research institutions in France or abroad, or from public or private research centers.
L'archive ouverte pluridisciplinaire HAL, est destinée au dépôt et à la diffusion de documents scientifiques de niveau recherche, publiés ou non, émanant des établissements d'enseignement et de recherche français ou étrangers, des laboratoires publics ou privés. 


\title{
A SOLUTION TO A PROBLEM OF D. LAU: COMPLETE CLASSIFICATION OF INTERVALS IN THE LATTICE OF PARTIAL BOOLEAN CLONES
}

\author{
MIGUEL COUCEIRO, LUCIEN HADDAD, KARSTEN SCHÖLZEL, \\ AND TAMÁS WALDHAUSER
}

This paper is dedicated to the distinguished scholar and friend Professor I.G. Rosenberg on the occasion of his 80th birthday.

\begin{abstract}
The following natural problem, first considered by D. Lau, has been tackled by several authors recently: Let $C$ be a total clone on $\mathbf{2}:=\{0,1\}$. Describe the interval $\mathcal{I}(C)$ of all partial clones on $\mathbf{2}$ whose total component is $C$. We establish some results in this direction and combine them with previous ones to show the following dichotomy result: For every total clone $C$ on $\mathbf{2}$, the set $\mathcal{I}(C)$ is either finite or of continuum cardinality.
\end{abstract}

\section{Preliminaries}

Let $k \geq 2$ be an integer and let $\mathbf{k}$ be a $k$-element set. Without loss of generality we assume that $\mathbf{k}:=\{0, \ldots, k-1\}$. For a positive integer $n$, an $n$-ary partial function on $\mathbf{k}$ is a map $f: \operatorname{dom}(f) \rightarrow \mathbf{k}$ where $\operatorname{dom}(f)$ is a subset of $\mathbf{k}^{n}$, called the domain of $f$. Let $\operatorname{Par}^{(n)}(\mathbf{k})$ denote the set of all $n$-ary partial functions on $\mathbf{k}$ and let $\operatorname{Par}(\mathbf{k}):=$ $\bigcup \operatorname{Par}^{(n)}(\mathbf{k})$. An $n$-ary partial function $g$ is said to be a total function if $\operatorname{dom}(g)=\mathbf{k}^{n}$. Let $\mathrm{Op}(\mathbf{k})$ be the set of all total functions on $\mathbf{k}$.

For every positive integer $n$ and each $1 \leq i \leq n$, let $e_{i}^{n}$ denote the $n$-ary $i$-th projection function defined by $e_{i}^{n}\left(a_{1}, \ldots, a_{n}\right)=a_{i}$ for all $\left(a_{1}, \ldots, a_{n}\right) \in \mathbf{k}^{n}$. Furthermore, let $J_{\mathbf{k}}:=\left\{e_{i}^{n} \mid 1 \leq i \leq n, n \in \mathbb{N} \backslash\{0\}\right\}$ be the set of all (total) projections.

Partial and total functions on $\mathbf{k}$ are composed in a natural way. We refer the reader to [4, 10, 11] for details.

Definition. A partial clone on $\mathbf{k}$ is a composition closed subset of $\operatorname{Par}(\mathbf{k})$ containing $J_{\mathbf{k}}$. If a partial clone is contained in the set of all total functions $\mathrm{Op}(\mathbf{k})$, then it is called a clone on $\mathbf{k}$.

Remark 1.1. There are several other equivalent definitions for partial clones. One definition uses Mal'cev's formalism and the other uses the concept of one point extension. These definitions can be found in [10] and in Chapter 20 of [1]. Later on in this paper we will use Mal'cev's elementary operations as described in [10] and [11].

\section{Example.}

1) For $a=0,1$ let $T_{a}$ be the set of all total functions satisfying $f(a, \ldots, a)=a$, $M$ be the set of all monotone total functions and $S$ be the set of all self-dual total functions on 2. Then $T_{0}, T_{1}, M$ and $S$ are clones on $\mathbf{2}$.

$2)$ Let $T_{0,2}:=\left\{f \in \mathrm{Op}(\mathbf{2}) \mid\left[\left(a_{1}, b_{1}\right) \neq(1,1), \ldots,\left(a_{n}, b_{n}\right) \neq(1,1)\right]\right.$

$T_{0,2}$ is a clone on 2 (see Chapter 3 of [1] for details.)

$$
\left.\Longrightarrow\left(f\left(a_{1}, \ldots, a_{n}\right), f\left(b_{1}, \ldots, b_{n}\right)\right) \neq(1,1)\right\} .
$$

3) Let $\widetilde{S}:=\left\{f \in \operatorname{Par}(\mathbf{2}) \mid\left\{\left(a_{1}, \ldots, a_{n}\right),\left(\neg a_{1}, \ldots, \neg a_{n}\right)\right\} \subseteq \operatorname{dom}(f)\right.$

where $\neg$ is the negation on 2 . Then $\widetilde{S}$ is a partial clone on $\mathbf{2}$.

$$
\left.\Longrightarrow f\left(\neg a_{1}, \ldots, \neg a_{n}\right)=\neg f\left(a_{1}, \ldots, a_{n}\right)\right\} \text {, }
$$

The idea behind the last two examples is formalized as follows. 
Definition. For $h \geq 1$ and $n \geq 1$, let $\rho$ be an $h$-ary relation on $\mathbf{k}$ and $f$ be an $n$-ary partial function on $\mathbf{k}$. We say that $f$ preserves $\rho$ if for every $h \times n$ matrix $M=\left[M_{i j}\right]$ whose columns $M_{* j} \in \rho,(j=1, \ldots, n)$ and whose rows $M_{i *} \in \operatorname{dom}(f)(i=1, \ldots, h)$, the $h$-tuple $\left(f\left(M_{1 *}\right), \ldots, f\left(M_{h *}\right)\right) \in \rho$. Define

$$
\operatorname{pPol} \rho:=\{f \in \operatorname{Par}(\mathbf{k}) \mid f \text { preserves } \rho\} .
$$

It is well known (see, e.g., [1] Chapter 20) that $\mathrm{pPol} \rho$ is a partial clone called the partial clone determined by the relation $\rho$.

Note that if there is no $h \times n$ matrix $M=\left[M_{i j}\right]$ whose columns $M_{* j} \in \rho$ and whose rows $M_{i *} \in \operatorname{dom}(f)$, then $f \in \operatorname{pPol} \rho$. Note also that the clone on $\mathbf{k}$ determined by the relation $\rho$ is $\operatorname{Pol} \rho:=\operatorname{pPol} \rho \cap \operatorname{Op}(\mathbf{k})$.

Thus in the example above $T_{0,2}=\operatorname{Pol}\{(0,0),(0,1),(1,0)\}$ and $\widetilde{S}=\operatorname{pPol}\{(0,1),(1,0)\}$.

All partial clones on $\mathbf{k}$ (clones on $\mathbf{k}$ ), ordered by inclusion, form a lattice $\mathcal{L}_{P_{\mathbf{k}}}\left(\mathcal{L}_{O_{\mathbf{k}}}\right.$, respectively) in which the infimum is the set-theoretical intersection. Clearly $\mathcal{L}_{O_{\mathbf{k}}}$ is a sublattice of $\mathcal{L}_{P_{\mathrm{k}}}$. It is therefore very natural to ask about the position of the lattice $\mathcal{L}_{O_{\mathbf{k}}}$ in $\mathcal{L}_{P_{\mathbf{k}}}$. In [12] D. Lau initiated the study of the following problem for the case $k=2$.

Problem. Let $C$ be a total clone on $\mathbf{2}:=\{0,1\}$. Describe the set of all partial clones on 2 whose total component is $C$, i.e., describe the set

$$
\begin{gathered}
\mathcal{I}(C):=\{D \subseteq \operatorname{Par}(\mathbf{2}) \mid D \text { is partial clone such that } \\
D \cap \operatorname{Op}(\mathbf{2})=C\} .
\end{gathered}
$$

The same question was asked for clones on the finite set $\mathbf{k}$ with $k \geq 2$, and results in this direction have been established recently, mainly concerning the maximal clones on k. We refer the reader to Section 20.7 in [1] for details. By Theorem 20.7.2 the set $\mathcal{I}(C)$ is an interval for every total clone $C$ on 2 .

In this paper we focus our attention to the case $k=2$. We give a full classification of all intervals $\mathcal{I}(C)$, where $C$ is one of the countably many clones on 2. More precisely, we first show that $\mathcal{I}\left(T_{0,2}\right)$ is of continuum cardinality on $\mathbf{2}$. Then we prove that the same result holds for any clone on $\mathbf{2}$ contained in one of $\left\{T_{0,2}, T_{1,2}\right\}$.

Finally we combine these results with previous known results discussed in [10] to prove our main result.

Let $\mathcal{F}:=\left\{\mathrm{Op}(\mathbf{2}), T_{0}, T_{1}, T_{0} \cap T_{1}, M, M \cap T_{0}, M \cap T_{1}, M \cap T_{0} \cap T_{1}, S, S \cap T_{0} \cap T_{1}\right\}$. We have:

Dichotomy Theorem. Let $C$ be a clone on $\mathbf{2}$. Then the interval $\mathcal{I}(C)$ in $\mathcal{L}_{P_{2}}$ of all partial clones whose total component is $C$ is finite if and only if $C \in \mathcal{F}$ and is of continuum cardinality otherwise.

We mention in passing that many results in this direction have been obtained by several authors, (see [1, 5, 6, 7, 8, 9, 10, 15, 16, 17]).

\section{Partial Clones intersecting $\mathrm{Op}(\mathbf{2})$ in $T_{0,2}$}

Let $\rho_{0,2}:=\{(0,0),(0,1),(1,0)\}$ and as seen above let $T_{0,2}:=\operatorname{Pol} \rho_{0,2}$. It is shown in 13] that the clone $T_{0,2}$ is covered by the clone $T_{0}=$ Pol $\{0\}$ of all 0 -preserving functions. In this section we construct a continuum family of partial clones on $\mathbf{2}$ whose intersection with $\mathrm{Op}(\mathbf{2})$ is $T_{0,2}$.

The idea behind the proof given in this section comes from [5] and is briefly discussed in [4].

Throughout let $k \geq 4$ be an even integer, and set $n(k)=k(k+1)+1$.

Define $R_{\uparrow}^{k}$ as the $n(k)$-ary relation whose members are tuples in which any two 1 's are separated by at least one 0 (in particular, the first and last positions cannot be simultaneously 1 , since we consider the indices modulo $n(k))$. For $i, j \in\{1, \ldots, n(k)\}$, we denote by $d(i, j)$ the circular distance between $i$ and $j$. 
Lemma 2.1. For every even integer $k \geq 4, T_{0,2} \subseteq \mathrm{pPol} R_{\uparrow}^{k}$.

Proof. Since

$$
R_{\uparrow}^{k}\left(x_{1}, \ldots, x_{n(k)}\right)=\bigwedge_{\substack{i, j \in[n(k)] \\ d(i, j)=1}} \varrho_{0,2}\left(x_{i}, x_{j}\right)
$$

we have, by the general theory (see e.g., the Representation Lemma 20.3.4 in 11]) that $\mathrm{pPol} \rho_{0,2} \subseteq \mathrm{pPol} R_{\uparrow}^{k}$, and since clearly $T_{0,2} \subseteq \mathrm{pPol} \rho_{0,2}$, the result follows.

Let $M_{\uparrow}^{k}$ be the $n(k) \times n(k)$ matrix with columns in $R_{\uparrow}^{k}$, the first being $c_{1}=$ $[1001010 \ldots 1010]^{T}$ and the remaining columns are obtained by applying cyclic shifts to $c_{1}$, i.e., $c_{2}=[01001010 \cdots 101]^{T}, c_{3}=[101001010 \cdots 10]^{T}, \ldots, c_{n(k)}=[001010 \cdots 101]^{T}$. Note that every entry on the diagonal of the matrix $M_{\uparrow}^{k}$ is 1 .

Remark 2.2. Let $r_{i}$ and $r_{j}$ be two rows of $M_{\uparrow}^{k}$. If $d(i, j) \geq 2$, then $r_{i}$ and $r_{j}$ have a 1 in the same position.

Lemma 2.3. If $k^{\prime}<k$, then there is no $n\left(k^{\prime}\right) \times n(k)$ matrix $N$ whose columns are in $R_{\uparrow}^{k^{\prime}}$ and whose rows are rows of $M_{\uparrow}^{k}$.

Proof. Suppose that $k^{\prime}<k$ and that $N$ is an $n\left(k^{\prime}\right) \times n(k)$ matrix whose columns are in $R_{\uparrow}^{k^{\prime}}$. For a contradiction, suppose that the rows of $N$ are rows of $M_{\uparrow}^{k}$. By Remark 2.2, the only possible "neighbor" rows of a row $r$ in $N$ are exactly the predecessor and successor rows of $r$ in $M_{\uparrow}^{k}$. But then $n\left(k^{\prime}\right)$ would be even, thus yielding the desired contradiction.

Define $R_{\downarrow}^{k}$ as the $n(k)$-ary relation whose members are tuples in which any two 1's are separated by at least $k 0$ 's (in particular, if the first position is 1 , then the last $k$ positions must be 0 ).

Lemma 2.4. For every even integer $k \geq 4, T_{0,2} \subseteq \mathrm{pPol} R_{\downarrow}^{k}$.

Proof. As in Lemma 2.1 since

$$
R_{\downarrow}^{k}\left(x_{1}, \ldots, x_{n(k)}\right)=\bigwedge_{\substack{i, j \in[n(k)] \\ 1 \leq d(i, j) \leq k}} \varrho_{0,2}\left(x_{i}, x_{j}\right)
$$

we have $T_{0,2} \subseteq \mathrm{pPol} \varrho_{0,2} \subseteq \mathrm{pPol} R_{\downarrow}^{k}$.

Let $M_{\downarrow}^{k}$ be the $n(k) \times n(k)$ matrix with columns in $R_{\downarrow}^{k}$, the first being $c_{1}^{\prime}=$ $[1 \underbrace{0 \cdots 0}_{k+1} 1 \underbrace{0 \cdots 0}_{k} \cdots 1 \underbrace{0 \cdots 0}_{k}]^{T}$ and the remaining columns are obtained by applying cyclic shifts to $c_{1}^{\prime}$ as before. As for the matrix $M_{\uparrow}^{k}$, every entry on the diagonal of $M_{\downarrow}^{k}$ is 1 .

Remark 2.5. Since $k \geq 4$ is even, if $r_{i}$ is a row of $M_{\uparrow}^{k}$, and $r_{j}^{\prime}$ is a row of $M_{\downarrow}^{k}$, then $r_{i}$ and $r_{j}^{\prime}$ have a 1 in the same position.

Lemma 2.6. If $k^{\prime}>k$, then there is no $n\left(k^{\prime}\right) \times n(k)$ matrix $N$ whose columns are in $R_{\downarrow}^{k^{\prime}}$ and whose rows are rows of $M_{\downarrow}^{k}$.

Proof. Suppose that $k^{\prime}>k$ and that $N$ is an $n\left(k^{\prime}\right) \times n(k)$ matrix whose columns are in $R_{\downarrow}^{k^{\prime}}$. For a contradiction, suppose that the rows of $N$ are rows of $M_{\downarrow}^{k}$. Since each row of $M_{\downarrow}^{k}$ has exactly $k 1$ 's, we have that $N$ has $k \cdot n\left(k^{\prime}\right) 1$ 's. Hence, $N$ has a column with at least $\frac{k \cdot n\left(k^{\prime}\right)}{n(k)} 1$ 's. However, it is easy to verify that since $k^{\prime}>k \geq 4$, we have that $\frac{k \cdot n\left(k^{\prime}\right)}{n(k)}>k^{\prime}$. But this yields the desired contradiction, since all columns of $N$ are members of $R_{\downarrow}^{k^{\prime}}$, and each has at most $k^{\prime} 1$ 's. 
Let $R_{k}$ be the $2 n(k)$-ary relation given by $R_{k}:=R_{\uparrow}^{k} \times R_{\downarrow}^{k}$. Since $T_{0,2} \subseteq \operatorname{pPol} R_{\uparrow}^{k}$ and $T_{0,2} \subseteq \mathrm{pPol} R_{\downarrow}^{k}$, we have $T_{0,2} \subseteq \mathrm{pPol} R_{k}$. Now as $T_{0,2} \subseteq \mathrm{pPol} R_{k}$, we have that $\mathrm{pPol} R_{k} \cap \mathrm{Op}(\mathbf{2})$ is one of $T_{0,2}, T_{0}$ or $\mathrm{Op}(\mathbf{2})$. As the $n(k)$-ary function $f$ on $\mathbf{2}$ defined by $f(0, \ldots, 0)=0$ and $f\left(x_{1}, \ldots, x_{n(k)}\right)=1$ if $\left(x_{1}, \ldots, x_{n(k)}\right) \neq(0, \ldots, 0)$ belongs to $T_{0}$ but does not preserve $R_{k}$, we conclude that $\mathrm{pPol} R_{k} \cap \mathrm{Op}(\mathbf{2})=T_{0,2}$, i.e., $\operatorname{pPol} R_{k} \in \mathcal{I}\left(T_{0,2}\right)$.

Define $M_{k}$ as the $2 n(k) \times n(k)$ matrix given by

$$
M_{k}=\left(\begin{array}{c}
M_{\uparrow}^{k} \\
M_{\downarrow}^{k}
\end{array}\right) .
$$

Note that each column of $M_{k}$ is a tuple of $R_{k}$.

Lemma 2.7. Let $N$ be a $2 n\left(k^{\prime}\right) \times n(k)$ matrix whose columns are in $R_{k^{\prime}}$ and whose rows are rows of $M_{k}$. Then, either all rows of $N$ are rows of $M_{\downarrow}^{k}$, or the first $n\left(k^{\prime}\right)$ are rows of $M_{\uparrow}^{k}$ and the remaining $n\left(k^{\prime}\right)$ are rows of $M_{\downarrow}^{k}$.

Proof. By Remark 2.2 and the fact that $R_{k^{\prime}}=R_{\uparrow}^{k^{\prime}} \times R_{\downarrow}^{k^{\prime}}$, not all of the last $n\left(k^{\prime}\right)$ rows can be rows of $M_{\uparrow}^{k}$, since for all columns in $R_{\downarrow}^{k^{\prime}}$ the distance between two 1 's is at least $k^{\prime}$. If we assume that there are rows from $M_{\uparrow}^{k}$ and $M_{\downarrow}^{k}$ at the same time, we see that two such rows are neighbors and by Remark 2.5 there is a column with adjacent 1's. But this contradicts the assumption that the columns belong to $R_{k^{\prime}}$. Thus there can only be rows from $M_{\downarrow}^{k}$ among the last $n\left(k^{\prime}\right)$ rows of $N$.

Moreover, from Remark 2.5 and the fact that $R_{k^{\prime}}=R_{\uparrow}^{k^{\prime}} \times R_{\downarrow}^{k^{\prime}}$, it follows that either all of the first $n\left(k^{\prime}\right)$ rows of $N$ are rows of $M_{\uparrow}^{k}$ or all of the first $n\left(k^{\prime}\right)$ rows of $N$ are rows of $M_{\downarrow}^{k}$.

Let $f_{k}$ be the $n(k)$-ary partial function whose domain is the set of rows of $M_{k}$, and such that $f_{k}$ is constant 1 on the rows of $M_{\uparrow}^{k}$ and constant 0 on the rows of $M_{\downarrow}^{k}$. Note that since both $M_{\uparrow}^{k}$ and $M_{\downarrow}^{k}$ have entries 1 on their diagonal, the partial function $f_{k}$ is undefined on the tuple $(0, \ldots, 0)$.

Lemma 2.8. Let $k, k^{\prime} \geq 4$ be even integers. Then $f_{k}$ preserves $R_{k^{\prime}}$ if and only if $k \neq k^{\prime}$.

Proof. Since $[1 \cdots 10 \cdots 0]^{T}$ does not belong to $R_{k^{\prime}}$, we have that if $k=k^{\prime}$, then $f_{k}$ does not preserve $R_{k^{\prime}}$.

So suppose that $k \neq k^{\prime}$. If $k<k^{\prime}$, then it follows from Lemmas 2.6 and 2.7, that $f_{k}$ preserves $R_{k^{\prime}}$ by default.

Suppose now that $k>k^{\prime}$. If $N$ is a $2 n\left(k^{\prime}\right) \times n(k)$ matrix whose columns are in $R_{k^{\prime}}$ and whose rows are rows of $M_{k}$ (otherwise we are done for the domain of $f_{k}$ is exactly the set of rows of $M_{k}$ ), then by Lemmas 2.3 and 2.7 it follows that all rows of $N$ are rows of $M_{\downarrow}^{k}$. Since $f_{k}$ is constant 0 on the rows of $M_{\downarrow}^{k}$, and since the $2 n\left(k^{\prime}\right)$-tuple all of whose entries are zero is a member of $R_{k^{\prime}}$, we conclude that $f_{k}$ preserves $R_{k^{\prime}}$.

Denote by $\mathbf{E}_{\geq 4}:=\{4,6,8, \ldots\}$ the set of all even integers greater than or equal to 4 and denote by $\mathcal{P}\left(\mathbf{E}_{\geq 4}\right)$ the power set of $\mathbf{E}_{\geq 4}$. Since $T_{0,2} \subseteq \operatorname{pPol} R_{k}$ for every even integer $k \geq 4$, we have that

$$
T_{0,2} \subseteq \bigcap_{k \in \mathbf{E}_{\geq 4} \backslash X} \operatorname{pPol} R_{k}
$$

for every subset $X$ of $\mathbf{E}_{\geq 4}$. By Lemma 2.8 the map

$$
\chi: \mathcal{P}\left(\mathbf{E}_{\geq 4}\right) \rightarrow \mathcal{I}\left(T_{0,2}\right) \cup \mathcal{I}\left(T_{0}\right) \cup \mathcal{I}(\mathrm{Op}(\mathbf{2}))
$$

defined by $X \longmapsto \chi(X):=\bigcap_{k \in \mathbf{E}_{>4} \backslash X} \operatorname{pPol} R_{k}$ is one-to-one. Since $\mathcal{I}\left(T_{0}\right)$ and $\mathcal{I}(\mathrm{Op}(\mathbf{2}))$ are finite (see Section 20.8 in [11]) we have the following result:

Theorem 2.9. The interval $\mathcal{I}\left(T_{0,2}\right)$ of partial clones on $\mathbf{2}$ is of continuum cardinality. 


\section{Partial Clones intersecting Op(2) in a subclone of $T_{0,2}$}

In this section we show that Theorem 2.9 holds for every subclone of $T_{0,2}$ in $\mathcal{L}_{\mathrm{O}_{2}}$. We will employ a result established in [10. First we need to recall some notations.

Let $f \in \operatorname{Par}(\mathbf{2})$ be $n$-ary and $g \in \operatorname{Par}(\mathbf{2})$ be $m$-ary. Then the superposition of $f$ and $g$, denoted $f \star g$ is the $(n+m-1)$-ary partial function on $\mathbf{2}$ defined by

$$
\begin{aligned}
\operatorname{dom}(f \star g):=\left\{\left(a_{1}, \ldots, a_{n+m-1}\right) \mid\right. & \left(a_{1}, \ldots, a_{m}\right) \in \operatorname{dom}(g) \text { and } \\
& \left.\left(g\left(a_{1}, \ldots, a_{m}\right), a_{m+1}, \ldots, a_{n+m-1}\right) \in \operatorname{dom}(f)\right\}
\end{aligned}
$$

and

$$
(f \star g)\left(a_{1}, \ldots, a_{n+m-1}\right):=f\left(g\left(a_{1}, \ldots, a_{m}\right), a_{m+1}, \ldots, a_{n+m-1}\right) .
$$

A set of partial functions $F \subseteq \operatorname{Par}(\mathbf{2})$ is called a closed set, if $F \star F \subseteq F, \zeta(F) \subseteq F$, $\tau(F) \subseteq F, \Delta(F) \subseteq F$ and $\nabla(F) \subseteq F$. The operations $\zeta, \tau, \Delta, \nabla, \star$ are known as Mal'cev's five elementary operations. We refer the reader to the introduction of 10 and to Section 20.1 of 11 for more details. Notice that it is well known that a set of partial functions $F \subseteq \operatorname{Par}(\mathbf{2})$ is a partial clone on $\mathbf{2}$ if and only if it is a closed subset and it contains the set of all projections $J_{2}$ (see, e.g., Section 20.1 of [1]).

We need the following result established in [10].

Lemma 3.1. (Theorem 8 [10]) Let $C$ be a clone over $\mathbf{2}$ and let $I$ be a nonempty set. Furthermore, let $\left(Q_{i}\right)_{i \in I}$ be a family of subsets of $\operatorname{Par}(\mathbf{2})$ such that

1) $Q_{i} \cap \operatorname{Op}(\mathbf{2})=\emptyset$,

2) $Q_{i}$ is a closed set of $\operatorname{Par}(\mathbf{2})$,

3) $Q_{i} \star C \subseteq Q_{i}$ and $C \star Q_{i} \subseteq Q_{i}$.

Then, for every subclone $B$ of $C$ on $\mathbf{2}$ and every $i \in I$, we have that $Q_{i} \cup B$ is a partial clone on 2. If furthermore $Q_{i} \neq Q_{j}$ for all $i, j \in I, i \neq j$, then $|\mathcal{I}(B)| \geq|I|$.

We use Theorem 2.9 and the lemma above to prove our second main result:

Theorem 3.2. Let $B \subseteq T_{0,2}$ be a clone on $\mathbf{2}$. Then the interval of partial clones $\mathcal{I}(B)$ is of continuum cardinality.

Proof. Denote by $U_{0}$ the set of all partial functions undefined on $(0, \ldots, 0)$, i.e.,

$$
U_{0}:=\{f \in \operatorname{Par}(\mathbf{2}) \mid(0, \ldots, 0) \notin \operatorname{dom}(f)\} .
$$

Now in Lemma 3.1 let $C$ be the clone $T_{0,2}$ on $\mathbf{2}, I$ be the set $\mathcal{P}\left(\mathbf{E}_{\geq 4}\right)$ as defined in the paragraph preceding Theorem 2.9, and for $X \in \mathcal{P}\left(\mathbf{E}_{\geq 4}\right)$ let $Q_{X}:=\left(\bigcap_{k \notin X} \operatorname{pPol} R_{k}\right) \cap U_{0}$. 3.1

We show that the family $\left(Q_{X}\right)_{X \in \mathcal{P}\left(\mathbf{E}_{>4}\right)}$ satisfies conditions 1), 2) and 3) of Lemma

1) Since $U_{0}$ contains no total functions we have $Q_{X} \cap \operatorname{Op}(\mathbf{2})=\emptyset$.

2) It is easy to verify that the sets $Q_{X}$ satisfy $\zeta\left(Q_{X}\right) \subseteq Q_{X}, \tau\left(Q_{X}\right) \subseteq Q_{X}, \Delta\left(Q_{X}\right) \subseteq$ $Q_{X}$ and $\nabla\left(Q_{X}\right) \subseteq Q_{X}$ (see, e.g., [10]). We show that $Q_{X} \star Q_{X} \subseteq Q_{X}$. Let $f, g \in Q_{X}$. Since $\bigcap_{k \notin X} \mathrm{pPol} R_{k}$ is a partial clone, we have that $f \star g \in \bigcap_{k \notin X} \mathrm{pPol} R_{k}$. Furthermore, since $(0, \ldots, 0) \notin \operatorname{dom}(g)$, we have that $(0, \ldots, 0) \notin \operatorname{dom}(f \star g)$, i.e., $f \star g \in U_{0}$ and thus $f \star g \in Q_{X}$. This shows that $Q_{X}$ is a closed set of $\operatorname{Par}(\mathbf{2})$.

3) To show that $Q_{X} \star T_{0,2} \subseteq Q_{X}$ take $f \in Q_{X}$ and $g \in T_{0,2}$. Since $T_{0,2} \subseteq$ pPol $R_{k}$ for all $k \geq 4$, we have $f \star g \in \bigcap_{k \notin X} \mathrm{pPol} R_{k}$ for every $X \in \mathcal{P}\left(\mathbf{E}_{\geq 4}\right)$ and it remains to show that $f \star g \in U_{0}$, i.e., $(0, \ldots, 0) \notin \operatorname{dom}(f \star g)$.

Indeed since $g \in T_{0,2} \subseteq T_{0}$, we have $g(0, \ldots, 0)=0$ and thus if $f \star g(0, \ldots, 0)=$ $f(g(0, \ldots, 0), 0, \ldots, 0)=f(0, \ldots, 0)$ was defined, then we would have $(0, \ldots, 0) \in$ dom $(f)$, a contradiction to $f \in U_{0}$. This gives that $f \star g \in U_{0}$ and so $f \star g \in Q_{X}$.

The proof of $T_{0,2} \star Q_{X} \subseteq Q_{X}$ is similar.

Now we show that $Q_{X} \neq Q_{Y}$ for every $X \neq Y, X, Y \in \mathcal{P}\left(\mathbf{E}_{\geq 4}\right)$. Since $X \neq Y$, say there is a $t \in X, t \notin Y$. Then by Lemma 2.8 $f_{t} \in \bigcap_{k \notin X} \operatorname{pPol} R_{k}$ and $f_{t} \notin \bigcap_{k \notin Y} \operatorname{pPol} R_{k}$. 


\begin{tabular}{|r|c|}
\hline$C$ & $|\mathcal{I}(C)|$ \\
\hline $\mathrm{Op}(\mathbf{2})$ & 3 \\
$T_{a}(a \in\{0,1\})$ & 6 \\
$M$ & 6 \\
$S$ & 6 \\
$T_{0} \cap T_{1}$ & 30 \\
$M \cap T_{a}(a \in\{0,1\})$ & 15 \\
$M \cap T_{0} \cap T_{1}$ & 101 \\
$S \cap T_{0} \cap T_{1}$ & 380 \\
\hline
\end{tabular}

TABLE 1. Sizes of the finite intervals $\mathcal{I}(C)$

It is shown in Section 2 that the partial functions $f_{k}$ are undefined on $(0, \ldots, 0)$, thus $f_{k} \in U_{0}$ for all $k \geq 4$. This shows that $f_{t} \in Q_{X}$ and $f_{t} \notin Q_{Y}$ proving that $Q_{X} \neq Q_{Y}$.

By Lemma 3.1 we have that $|\mathcal{I}(B)| \geq\left|\mathcal{P}\left(\mathbf{E}_{\geq 4}\right)\right|$ and thus $\mathcal{I}(B)$ is of continuum cardinality. have:

Now let $\rho_{1,2}:=\{(0,1),(1,0),(1,1)\}$ and let $T_{1,2}:=\operatorname{Pol} \rho_{1,2}$. Then by duality we

Theorem 3.3. Let $B \subseteq T_{1,2}$ be a clone on $\mathbf{2}$. Then the interval of partial clones $\mathcal{I}(B)$ is of continuum cardinality.

\section{Complete classification of all intervals of the form $\mathcal{I}(C)$}

In this section we use results discussed in [10] and combine them with our results established in the previous sections to complete the classification of all intervals of partial clones of the form $\mathcal{I}(C)$ over 2 . Let $T_{a}$ (for $a \in\{0,1\}$ ), $M$ and $S$ be as defined in Section 1. Let $L$ be the clone of all linear functions, furthermore for $a \in\{0,1\}$ and $\mu \geq 2$ let $T_{a, \mu}=\operatorname{Pol}\left(\{0,1\}^{\mu} \backslash\{(\neg a, \ldots, \neg a)\}\right)$ and $T_{a, \infty}=\bigcap_{\mu \geq 2} T_{a, \mu}, \Lambda$ be the clone generated by $\left\{\wedge, c_{0}, c_{1}\right\}$ and $V$ be the clone generated by $\left\{\vee, c_{0}, c_{1}\right\}$ on 2 .

Set $\mathcal{F}:=\left\{\operatorname{Op}(\mathbf{2}), T_{0}, T_{1}, T_{0} \cap T_{1}, M, M \cap T_{0}, M \cap T_{1}, M \cap T_{0} \cap T_{1}, S, S \cap T_{0} \cap T_{1}\right\}$.

In [10] the authors collect several known results and establish some new ones concerning the intervals $\mathcal{I}(C)$ where $C$ is a clone on $\mathbf{2}$. The following is a conclusion of [10].

Theorem 4.1. Let $C$ be a clone on 2. Then the interval of partial clones $\mathcal{I}(C)$ over 2 is finite if and only if $C \in \mathcal{F}$. Furthermore if $C \subseteq B$ with $B \in\left\{L, \Lambda, V, T_{0, \infty}, T_{1, \infty}\right\}$, then the set $\mathcal{I}(C)$ has the cardinality of the continuum. Finally if $C \subseteq B$ with $B \in$ $\left\{T_{0,2}, T_{1,2}\right\}$, then the set $\mathcal{I}(C)$ is infinite.

The reader can verify that with the exception of subclones of $T_{0, \infty}$ and $T_{1, \infty}$, the theorem above leaves open the cardinality of $\mathcal{I}(C)$ for almost all subclones $C$ of $T_{0,2}$ and $T_{1,2}$ (see Figure 1 at the end of this section for the positions of these various clones on 2 in the Post Lattice).

Combining Theorems 3.2, 3.3 and 4.1 gives our Dichotomy Theorem stated in Section 1 of this paper.

We mention in passing that as the clone $S \cap M$ is a subclone of $T_{0,2}$, we have by Theorem 3.2 that the interval of partial clones $\mathcal{I}(S \cap M)$ is of continuum cardinality. A result in this direction is shown in 5] where a continuum family of partial clones containing the set of all partial monotone and self-dual functions is constructed.

Remark 4.2. This paper shows that there is no interval of partial clones of the form $\mathcal{I}(C)$ that is countably infinite in $\mathcal{L}_{P_{\mathbf{2}}}$. 


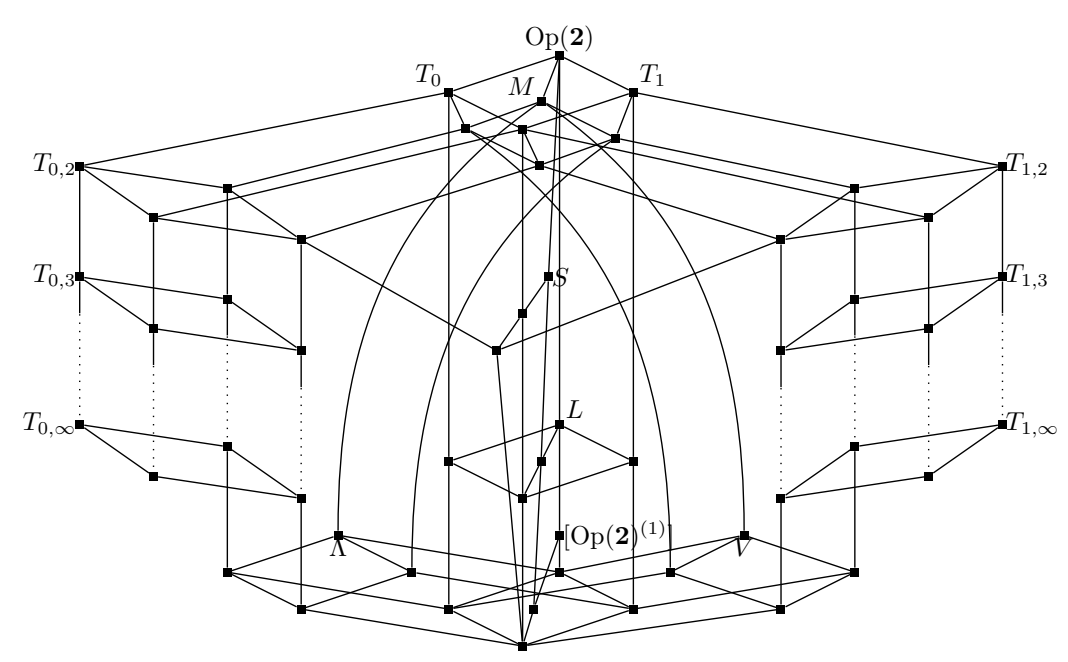

Figure 1. Post's lattice

Call a partial clone $D$ on $\mathbf{2}$ strong if it contains all subfunctions of its functions, i.e., if for every $g \in \operatorname{Par}(\mathbf{2})$, we have $g \in D$ whenever $g=\left.f\right|_{\operatorname{dom}(g)}$, for some $f \in D$. Now the lattice $\mathcal{L}_{\mathrm{O}_{2}}$ is a countably infinite sublattice of $\mathcal{L}_{\mathrm{P}_{2}}$, but $\mathcal{L}_{\mathrm{O}_{2}}$ consists of clones of total functions only. Thus the clones in $\mathcal{L}_{\mathrm{O}_{2}}$ are not strong partial clones on $\mathbf{2}$.

We pose the following problem:

Problem. Does the lattice $\mathcal{L}_{P_{2}}$ have a countably infinite interval of strong partial clones?

Note added in proof. One year after this paper was written, several such examples were constructed, see [3].

Remark 4.3. As defined above let $\rho_{0,2}:=\{(0,0),(0,1),(1,0)\}$ and let $T_{0,2}:=$ Pol $\rho_{0,2}$. Theorem 2.9 says that the interval of all partial clones that intersect $\mathrm{Op}(\mathbf{2})$ in $T_{0,2}$ is of continuum cardinality over 2. More results in this direction are established in [2]. Let $\left\langle\rho_{0,2}\right\rangle$ be the smallest closed class of relations (see [11], Section 2.4) that contain $\rho_{0,2}$ and let $\mathcal{G}$ be the set of all undirected finite graphs without multiple edges but possibly with loops (up to an isomorphism). An appropriate closure operator is introduced on $\mathcal{G}$ in 2 such that the closed classes of graphs are in a one-to-one correspondence with the closed subclasses of $\left\langle\rho_{0,2}\right\rangle$ which in turn are in a one-to-one correspondence with the strong partial clones containing $T_{0,2}$. This gives a simple proof to the result established in Theorem 2.9 in the present paper. Moreover, this correspondence allows us to to give some interesting descriptions of the bottom and the top of the lattice of all strong partial clones containing the total clone $T_{0,2}$ on $\mathbf{2}$.

\section{ACKnowledgments}

The authors thank the anonymous referees for their valuable comments on the previous version of this paper. L. Haddad is supported by Academic Research Program of RMC. K. Schölzel is supported by the internal research project MRDO2 of the University of Luxembourg. T. Waldhauser acknowledges that the present project is supported by the Hungarian National Foundation for Scientific Research under grants no. K83219 and K104251. The present project is supported by the European Union and co-funded by the European Social Fund under the project "Telemedicine-focused research activities on the field of Mathematics, Informatics and Medical sciences" of project number "TÁMOP-4.2.2.A-11/1/KONV-2012-0073". 


\section{REFERENCES}

[1] V. B. Alekseev and L. L. Voronenko. On some closed classes in partial two-valued logic. Diskretn. Mat. 6(4) (1994) 58-79 (Russian). English translation: Discrete Math. Appl., 4(5) (1994) 401419.

[2] M. Couceiro, L. Haddad, E. Lehtonen, K. Schölzel and T. Waldhauser. Relation graphs and partial clones on a 2-element set. Proc. 44th IEEE International Symposium on Multiple-Valued Logic, pp 161-166, Bremen, Germany, May 2014.

[3] L. Haddad and K. Schölzel. Countable Intervals of Partial Clones. Proc. 44th IEEE International Symposium on Multiple-Valued Logic, pp 155-160, Bremen, Germany, May 2014.

[4] M. Couceiro and L. Haddad. A survey on intersections of maximal partial clones of Boolean partial functions. Proc. 42nd IEEE International Symposium on Multiple-Valued Logic, pp 287292, Victoria, Canada, May 2012.

[5] M. Couceiro, L. Haddad and I. G. Rosenberg, Partial clones containing all Boolean monotone self-dual partial functions. Preprint 2011

[6] J. Fugère and L. Haddad. On Partial Clones Containing All Idempotent Partial Operations. Proc. 28th IEEE International Symposium on Multiple-Valued Logic, pp 369-373, Fukuoka, Japan, May 1998.

[7] L. Haddad and I. G. Rosenberg. Partial clones containing all permutations. Bull. Aust. Math. Soc. 52, No. 2, pp 263-278 (1995).

[8] L. Haddad and G. E. Simons. On intervals of partial clones of Boolean partial functions. Proc. 33rd IEEE International Symposium on Multiple-Valued Logic, pp 315-320, Tokyo, Japan, May 2003.

[9] L. Haddad and G. E. Simons, Interval of Boolean Partial Clones. Italian Journal of Pure and App. Math. , No. 21 (2007), pp 147-162.

[10] D. Lau and K. Schölzel, A Classification of Partial Boolean Clones. Proc. 40th IEEE International Symposium on Multiple-Valued Logic, pp 189 - 194, Barcelona, Spain, May 2010.

[11] D. Lau, Function Algebras on Finite Sets, a basic course on Multiple-Valued Logic and Clone Theory, 670 pages, Springer Monograph in Mathematics, 2006.

[12] D. Lau, Über partielle Funktionenalgebren. Rostock. Math. Kolloq. 33 (1988), pp 23-48.

[13] E. Post, The two-valued iterative systems of mathematical logic, Ann. Math. Studies 5. Princeton Univ. Press 1941.

[14] B. A. Romov. The algebras of partial functions and their invariants. Kibernetika 2 (1981) 1-11 (Russian). English translation: Cybernetics 17 (1981) 157-167.

[15] B. Strauch. Die Menge $\mathcal{M}\left(M \cap T_{0} \cap T_{1}\right)$. Preprint Universität Rostock (1995).

[16] B. Strauch. Die Menge $\mathcal{M}\left(S \cap T_{0} \cap T_{1}\right)$. Preprint Universität Rostock (1996).

[17] B. Strauch. On partial classes containing all monotone and zero-preserving total Boolean functions. Math. Log. Quart. 43 (1997) 510-524.

[18] B. Strauch. Noncountable many classes containing a fixed class of total Boolean functions. General Algebra and applications in Discrete Mathematics, Proceedings of "Conference on General Algebra and Discrete Mathematics" (edited by K. Denecke and O. Lüders), Shaker Verlag, Aachen (1997) 177-188.

(M. Couceiro) LAMSAdE - CNRS, Université Paris-Dauphine, Place du Maréchal de Lattre de Tassigny, 75775 Paris Cedex 16, France and LORIA (CNRS - Inria Nancy Grand Est - Université de Lorraine), Equipe Orpailleur - BÂtiment B, Campus Scientifique, B.P. 239, 54506 VANDeEuvre-LÈS-NANCy CEDEX, France

E-mail address: miguel.couceiro@inria.fr

(L. Haddad) Department of Mathematics and Computer Science, Royal Military College of Canada, PO Box 17000, Station Forces, Kingston, Ontario, K7K 7B4 Canada

E-mail address: haddad-1@rmc.ca

(K. Schölzel) Mathematics Research Unit, University of Luxembourg, 6, Rue Richard Coudenhove-Kalergi, L-1359 Luxembourg, Luxembourg

E-mail address: dr.karsti@gmail.com

(T. Waldhauser) Bolyai Institute, University of Szeged, Aradi vértanúk tere 1, H-6720 SzEgEd, Hungary

E-mail address: twaldha@math.u-szeged.hu 